\title{
Oscillatory Free Convection about a Horizontal Circular Cylinder in the Presence of Heat Generation
}

\author{
Najahulfazliah Zainuddin, ${ }^{1}$ Muhaimin Ismoen, ${ }^{1}$ Rozaini Roslan, ${ }^{1}$ and Ishak Hashim ${ }^{2,3}$ \\ ${ }^{1}$ Centre for Research in Computational Mathematics, Faculty of Science, Technology and Human Development, \\ Universiti Tun Hussein Onn, 86400 Batu Pahat, Johor, Malaysia \\ ${ }^{2}$ Centre for Modelling \& Data Analysis, School of Mathematical Sciences, Universiti Kebangsaan Malaysia (UKM), \\ 43600 Bangi, Selangor, Malaysia \\ ${ }^{3}$ Department of Mathematics, Faculty of Science, King Abdulaziz University, P.O. Box 80257, Jeddah 21589, Saudi Arabia
}

Correspondence should be addressed to Ishak Hashim; ishak_h@ukm.my

Received 4 February 2014; Accepted 31 March 2014; Published 22 April 2014

Academic Editor: Mohamed Abd El Aziz

Copyright (C) 2014 Najahulfazliah Zainuddin et al. This is an open access article distributed under the Creative Commons Attribution License, which permits unrestricted use, distribution, and reproduction in any medium, provided the original work is properly cited.

\begin{abstract}
The oscillatory free convection about a horizontal circular cylinder in a Newtonian fluid in the presence of heat generation is investigated numerically by using the finite difference method. The surface temperature of the cylinder oscillates harmonically about the temperature of the surrounding medium. Heat is generated internally within the Newtonian fluid at a rate proportional to a power of the temperature difference. It is found that the presence of heat generation significantly increases the temperature and velocity distribution. The effects of the heat generation parameter and the Prandtl number on the surface rate of heat transfer, in terms of the local Nusselt number, and the surface shear stress, in terms of the local skin friction, are shown graphically from the stagnation point of the circular cylinder.
\end{abstract}

\section{Introduction}

The study of oscillatory free convective flow is very important in the engineering field of fluid mechanics. This is because such flows often occur in practice, while the heat generation is important for physical problems. Much information has been obtained about free convection over a circular cylinder, but less has been found about when this takes place in the presence of heat generation. Besides that, there are many related problems with the presence of heat generation but where the surface temperature is uniformly constant or steady. Vajravelu and Hadjinicolaou [1] presented heat transfer characteristics in a laminar boundary layer flow of viscous fluid over a linearly stretching continuous surface with viscous dissipation or frictional heating and internal heat generation. In this study, they considered the volumetric rate of heat generation, $q^{\prime \prime \prime}\left[\mathrm{W} / \mathrm{m}^{3}\right]$, to be

$$
q^{\prime \prime \prime}= \begin{cases}Q_{0}\left(T-T_{\propto}\right), & \text { for } T \geq T_{\propto}, \\ 0, & \text { for } T<T_{\propto},\end{cases}
$$

where $Q_{0}$ is the heat generation constant. The above relation is valid for the state of some exothermic processes having $T_{\propto}$ as the onset temperature. This study was extended by Vajravelu [2] to natural convection on a heated semi-infinite vertical plate with internal heat generation. The effects of heat generation over a plate, a cylinder, and a sphere have been investigated by researchers. The work conducted by Vajravelu and Hadjinicolaou [1] has been continued by other researchers. Chamkha and Issa [3] studied the effects of heat generation or absorption on a hydromagnetic flow over a flat surface, while Molla et al. [4] studied the natural convection flow along a vertical wavy surface with a uniform surface temperature in the presence of heat generation or absorption. Meanwhile, Ferdousi and Alim [5] considered the effect of heat generation on natural convection flow from a porous vertical plate.

Some works have also appeared on natural convection in the presence of heat generation over a horizontal circular cylinder. Molla et al. [6] studied an isothermal horizontal circular cylinder, and this work was extended in [7] to 
the existence of uniform heat flux. Molla et al.s work $[6,7]$ was reviewed by Cheng [8] in relation to the study of a natural convection boundary layer on a horizontal elliptical cylinder with constant heat flux and internal heat generation, while the effect of radiation on a natural convection flow over a sphere in the presence of heat generation was investigated by Miraj et al. [9].

Unsteady free convection about a circular cylinder where the temperature of the surface either oscillates or is constant was studied in application technology. The oscillatory circular cylinder problem can be solved either analytically or numerically. Merkin [10] was the first who studied this related problem in oscillatory free convection for an infinite horizontal cylinder. He solved it analytically by using a matching technique between the inner and outer boundary layers. Continuing the work of Merkin [10], Chatterjee and Debnath [11] solved the problem by using the technique of separating the flow field into a steady and an unsteady part from the beginning in the governing equations. Ganesan and Rani [12] presented the problem of transient natural convection along a vertical cylinder with heat and mass transfer and solved it by using the implicit finite difference Crank-Nicolson scheme that had been used by Rani and Devaraj [13] in their study of the unsteady flow past a vertical cylinder with temperature oscillations. Jaman and Hossain [14] investigated the effect of fluctuating surface temperature on natural convection flow over cylinders of elliptic cross section and solved the problem by the most efficient and accurate implicit finite difference method together with the Keller-box elimination technique (also known as the Kellerbox method).

Some work on free convection over other types of geometric shapes was carried out by Verma and Singh [15], who studied the effect of plate oscillations on horizontal free convection flow. Meanwhile, Hossain et al. [16] presented the heat transfer response of MHD free convection flow from a vertical heated plate to an oscillating surface heat flux. The solutions for general frequencies were obtained using the Keller-box method. The partial differential equations were reduced to a pair of linear ordinary differential equations and the solutions were obtained using a straightforward shooting method. A study of unsteady free convection heat and mass transfer in Walters-B viscoelastic flow past a semi-infinite vertical plate has been carried out by Prasad et al. [17] using an efficient, accurate, and implicit finite difference scheme of the Crank-Nicolson type. This method was originally developed by Prasad et al. [18] in their study of radiation and mass transfer effects on two-dimensional flow past an impulsively started infinite vertical plate. Udin and Kumar [19] studied unsteady free convection in a fluid past an inclined plate immersed in a porous medium numerically by using explicit finite difference, as demonstrated in Carnahan et al. [20]. Recently, Kasim et al. [21] considered the unsteady free convection flow over a three-dimensional stagnation point with internal heat generation or absorption; the governing equations were solved by an implicit finite difference method.

Oscillatory free convection about a circular cylinder in the presence of heat generation is new and has not yet been studied. The governing equations and boundary equations

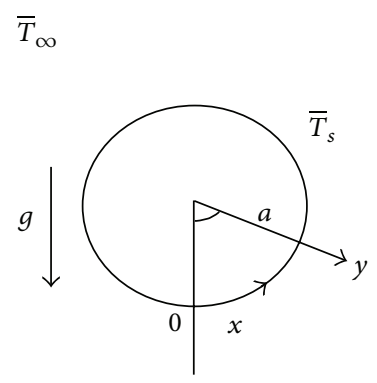

Figure 1

are first transformed into a dimensionless form. The resulting system of equations is solved by a finite difference CrankNicolson scheme.

\section{Formulation of Problem}

Two-dimensional oscillatory free convection on a circular cylinder of radius $a$ in presence of heat generation $\gamma$ which is aligned in an ambient fluid of temperature $\bar{T}_{\infty}$ is considered. The physical configuration considered is as shown in Figure 1. The surface temperature of the cylinder is assumed to be $\bar{T}_{s}$ where $\bar{T}_{s}>\bar{T}_{\infty}$. Extending the work of Molla et al. [6] and Merkin [10], who considered the effect of heat generation on free convection, and oscillatory free convection on a cylinder, respectively, the equations for mass continuity, momentum, and energy are governed by

$$
\begin{gathered}
\frac{\partial u}{\partial x}+\frac{\partial v}{\partial y}=0 \\
\frac{\partial u}{\partial \bar{t}}+u \frac{\partial u}{\partial x}+v \frac{\partial u}{\partial y}=v\left(\frac{\partial^{2} u}{\partial y^{2}}\right)+g \beta\left(\bar{T}-\bar{T}_{\infty}\right) \sin \left(\frac{x}{a}\right), \\
\frac{\partial \bar{T}}{\partial \bar{t}}+u \frac{\partial \bar{T}}{\partial x}+v \frac{\partial \bar{T}}{\partial y}=\frac{k}{\rho C_{\rho}}\left(\frac{\partial^{2} \bar{T}}{\partial y^{2}}\right)+\frac{Q_{0}}{\rho C_{\rho}}\left(\bar{T}-\bar{T}_{\infty}\right),
\end{gathered}
$$

where $x$ is measured along the surface of the circular cylinder and $y$ is measured normal to the surface while $\bar{t}$ is the time and $u$ and $v$ denote the velocity components in the $x$ and $y$ directions, respectively. The gravitational acceleration $g$ acts downward and $\beta$ is the coefficient of thermal expansion. $(x / a)$ is the angle of the $y$-axis. $\bar{T}$ is the temperature of fluid and $\bar{T}_{\infty}$ is the temperature that is far away from the cylinder. $k$ is the thermal conductivity, $\rho$ is the fluid density, $C_{\rho}$ is the specific heat at constant pressure, and $Q_{0}$ is a heat generation constant. The boundary conditions are defined as follows:

$$
\begin{gathered}
u=v=0, \quad \bar{T}=\left(\bar{T}_{s}-\bar{T}_{\infty}\right) \cos \omega \bar{t}+\bar{T}_{s} \quad \text { at } y=0, \\
u \longrightarrow 0, \quad \bar{T} \longrightarrow \bar{T}_{\infty} \quad \text { at } y \longrightarrow \infty .
\end{gathered}
$$


Here $\bar{T}_{s}$ is the temperature of the cylinder. $\omega$ is the frequency parameter. The above equations become dimensionless by substituting the following nondimensional variables:

$$
\begin{aligned}
& X=\frac{x}{a}, \quad Y=\frac{y}{a} \mathrm{Gr}^{1 / 4}, \quad \bar{t}=\frac{v}{a^{2}} \mathrm{Gr}^{1 / 2} t, \\
& T=\frac{\left(\bar{T}-\bar{T}_{\infty}\right)}{\left(\bar{T}_{s}-\bar{T}_{\infty}\right)}, \quad U=\frac{a}{v} \mathrm{Gr}^{1 / 2} u, \\
& V=\frac{a}{v} \mathrm{Gr}^{-1 / 4} v, \quad \mathrm{Gr}=g \beta \frac{\left(\bar{T}_{s}-\bar{T}_{\infty}\right)}{v^{2}} a^{3}, \\
& v=\frac{\mu}{\rho}, \quad \operatorname{Pr}=\frac{\mu C_{\rho}}{k}, \quad \gamma=\frac{Q_{0} a^{2}}{\mu C_{\rho} \mathrm{Gr}^{1 / 2}},
\end{aligned}
$$

where $v$ is the reference kinematic viscosity, Gr is the Grashof number, Pr is the Prandtl number, and $\gamma$ is the heat generation parameter. By substituting the variables (4) into (2), the equations are reduced to the following form:

$$
\begin{gathered}
\frac{\partial U}{\partial X}+\frac{\partial V}{\partial Y}=0 \\
\frac{\partial U}{\partial t}+U \frac{\partial U}{\partial X}+V \frac{\partial U}{\partial Y}=\frac{\partial^{2} U}{\partial Y^{2}}+T \sin X \\
\frac{\partial T}{\partial t}+U \frac{\partial T}{\partial X}+V \frac{\partial T}{\partial Y}=\frac{1}{\operatorname{Pr}} \frac{\partial^{2} T}{\partial Y^{2}}+\gamma T
\end{gathered}
$$

By assuming $\tau=\omega \bar{t}$, the corresponding initial and boundary conditions in dimensionless form are as follows:

$$
\begin{gathered}
U=V=0, \quad T=1+\cos \tau \quad \text { at } Y=0, \\
U \longrightarrow 0, \quad T \longrightarrow T_{0} \quad \text { as } Y \longrightarrow \infty .
\end{gathered}
$$

By using the nondimensional quantities (4), the surface rate of heat transfer in terms of the local Nusselt number,and the surface shear stress in terms of the local skin friction are as follows:

$$
\begin{gathered}
C_{f} \mathrm{Gr}^{1 / 4}=X\left(\frac{\partial U}{\partial Y}\right)_{Y=0}, \\
\mathrm{NuGr}^{-1 / 4}=-\left(\frac{\partial T}{\partial Y}\right)_{Y=0} .
\end{gathered}
$$

\section{Solution Procedure}

The nonlinear partial differential equations (5) and (6) under the initial and boundary conditions (8) are solved numerically by an implicit finite difference Crank-Nicolson scheme. The region of integration is considered where an axis along the surface $X_{\max }=\pi$ and an axis normal to the surface $Y_{\max }=30.0$ corresponding to $Y_{\infty}$. There are $M$ and $N$ grid spacing in the $X$ and $Y$ directions, respectively. The mesh points in the $(X, Y)$ plane by $X_{i}$ and $Y_{j}$, where $i=0,1,2,3, \ldots, M$ and $j=0,1,2,3, \ldots, N$. The mesh sizes have been fixed as $\Delta X=\pi / 150, \Delta Y=0.12$ with time step $\Delta \tau=0.01$. The iterative procedure is stopped when the time steps reach $\tau_{\max }=30$. The numerical values of the variables such as $U, V$ and the $T$ temperature are obtained at specific points. The finite difference equations corresponding to (5), (6) and (7) are as follows:

$$
\begin{aligned}
& \frac{1}{2}\left(\frac{V_{i, j}^{k+1}-V_{i, j-1}^{k+1}}{\Delta Y}+\frac{V_{i, j}^{k}-V_{i, j-1}^{k}}{\Delta Y}\right) \\
& =\frac{1}{2}\left(\frac{U_{i, j}^{k+1}-U_{i-1, j}^{k+1}}{\Delta X}+\frac{U_{i, j}^{k}-U_{i-1, j}^{k}}{\Delta X}\right), \\
& \frac{U_{i, j}^{k+1}-U_{i, j}^{k}}{\Delta t}+\frac{1}{2}\left[U_{i, j}^{k}\left(\frac{U_{i, j}^{k+1}-U_{i-1, j}^{k+1}+U_{i, j}^{k}-U_{i-1, j}^{k}}{\Delta X}\right)\right. \\
& \left.+V_{i, j}^{k}\left(\frac{U_{i, j}^{k+1}-U_{i, j-1}^{k+1}+U_{i, j}^{k}-U_{i, j-1}^{k}}{\Delta Y}\right)\right] \\
& =\frac{1}{2}\left[\left(\frac{U_{i, j+1}^{k+1}-2 U_{i, j}^{k+1}+U_{i, j-1}^{k+1}+U_{i, j+1}^{k}-2 U T_{i, j}^{k}+U_{i, j-1}^{k}}{\Delta Y^{2}}\right)\right. \\
& \left.-\gamma\left(T_{i, j}^{k+1}+T_{i, j}^{k}\right) \sin X_{i}\right] \\
& \frac{T_{i, j}^{k+1}-T_{i, j}^{k}}{\Delta t}+\frac{1}{2}\left[U_{i, j}^{k}\left(\frac{T_{i, j}^{k+1}-T_{i-1, j}^{k+1}+T_{i, j}^{k}-T_{i-1, j}^{k}}{\Delta X}\right)\right. \\
& \left.+V_{i, j}^{k}\left(\frac{T_{i, j}^{k+1}-T_{i, j-1}^{k+1}+T_{i, j}^{k}-T_{i, j-1}^{k}}{\Delta Y}\right)\right] \\
& =\frac{1}{2}\left[\frac{1}{\operatorname{Pr}}\left(\frac{T_{i, j+1}^{k+1}-2 T_{i, j}^{k+1}+T_{i, j-1}^{k+1}+T_{i, j+1}^{k}-2 T_{i, j}^{k}+T_{i, j-1}^{k}}{\Delta Y^{2}}\right)\right. \\
& \left.+\gamma\left(T_{i, j}^{k+1}+T_{i, j}^{k}\right)\right]
\end{aligned}
$$

The coefficients $U_{i, j}^{k}$ and $V_{i, j}^{k}$ in (11) and (12) are treated as constant at any one time step. At time $\tau=0$, the values of $T_{i, j}^{k}, U_{i, j}^{k}$, and $V_{i, j}^{k}$ are known while the values of $T_{i, j}^{k+1}, U_{i, j}^{k+1}$, and $V_{i, j}^{k+1}$ for the next time step are unknown and calculated by using Crank-Nicolson method.

\section{Results and Discussion}

The effect of heat generation on free convection on a circular cylinder with oscillating surface temperature was investigated. Solutions were obtained for several Prandtl numbers, $\operatorname{Pr}=0.7,2.0,3.0,7.0$ and different values of the heat generation parameter $\gamma=0.0,0.2,0.4,0.6,0.8$. 
TABLE 1: Temperature values when $\tau=6.00$ at different $M \times N$ with $\gamma=0.2$ and $\operatorname{Pr}=0.7$.

\begin{tabular}{ccccc}
\hline & $X$ & $50 \times 150$ & $100 \times 200$ & $150 \times 250$ \\
& 0 & 0.3713 & 0.3985 & 0.4159 \\
& $\pi / 6$ & 0.5041 & 0.5429 & 0.5652 \\
& $\pi / 3$ & 0.5432 & 0.5764 & 0.5992 \\
& $\pi / 2$ & 0.6011 & 0.6361 & 0.6571 \\
& $2 \pi / 3$ & 0.6863 & 0.7266 & 0.7447 \\
& $5 \pi / 6$ & 0.8229 & 0.8468 & 0.8688 \\
& $\pi$ & 0.9910 & 1.0224 & 1.0397 \\
\hline
\end{tabular}

TABLE 2: Comparison of the numerical results in the present study for the rate of heat transfer $\mathrm{NuGr}^{-1 / 4}$ and the local skin friction coefficients $C_{f} \mathrm{Gr}^{1 / 4}$ with those of Molla et al. [6] for $\gamma=0.0$ and $\operatorname{Pr}=1.0$.

\begin{tabular}{lcccc}
\hline & \multicolumn{2}{c}{$\mathrm{NuGr}^{-1 / 4}$} & \multicolumn{2}{c}{$C_{f} \mathrm{Gr}^{1 / 4}$} \\
$X$ & $\begin{array}{c}\text { Present } \\
\text { study }\end{array}$ & $\begin{array}{c}\text { Molla et al. } \\
{[6]}\end{array}$ & $\begin{array}{c}\text { Present } \\
\text { study }\end{array}$ & $\begin{array}{c}\text { Molla et al. } \\
{[6]}\end{array}$ \\
\hline 0 & 0.4221 & 0.4241 & 0.0000 & 0.0000 \\
$\pi / 6$ & 0.4193 & 0.4161 & 0.4064 & 0.4145 \\
$\pi / 3$ & 0.4050 & 0.4005 & 0.7392 & 0.7539 \\
$\pi / 2$ & 0.3810 & 0.3740 & 0.9562 & 0.9541 \\
$2 \pi / 3$ & 0.3306 & 0.3355 & 0.9668 & 0.9696 \\
$5 \pi / 6$ & 0.2481 & 0.2812 & 0.7523 & 0.7739 \\
$\pi$ & 0.1975 & 0.1917 & 0.3245 & 0.3264 \\
\hline
\end{tabular}

A grid independence test was carried out when $\tau=6.00$ with $\gamma=0.2$ and $\operatorname{Pr}=0.7$ by using sets of grids in the range of $50 \times 150,100 \times 200$, and $150 \times 250$ mesh points. Table 1 shows that there are insignificant differences between sets of grids with $150 \times 250$ mesh points and above. Therefore, for all computations of similar problems in this section a $150 \times 250$ grid will be used.

For the steady state, the comparison of the numerical results of the present study with those of Molla et al. [6] is discussed in Table 2 without the effects of heat generation $(\gamma=0.0)$ and with Prandtl number $\operatorname{Pr}=1.0$. The results obtained start from the lower stagnation point of the circular cylinder $X=0$ and go up to the upper stagnation point $X_{i}=\pi$ on the surface of the circular cylinder. The results of the present study and those of Molla et al. [6] are found to be in excellent agreement.

The temperature and velocity profiles are shown against the axial coordinate $X$ at different values of the radial coordinate $Y$. Here, the numerical results are those obtained at time $\tau=6.00$ with $\gamma=0.2$ and $\operatorname{Pr}=0.7$. In Figure 2, the temperature profile increases with $X$. It is proved that the increasing values of $X$ from the lower stagnation point of the circular cylinder $X=0$ to the upper stagnation point $X_{i}=\pi$ lead to an increase in the temperature profile. In addition, the temperature profiles near the surface are higher, and they decrease as the distance from the surface increases. The velocity profiles shown in Figure 3 increase from the surface of the cylinder and decrease for some value of $Y$.

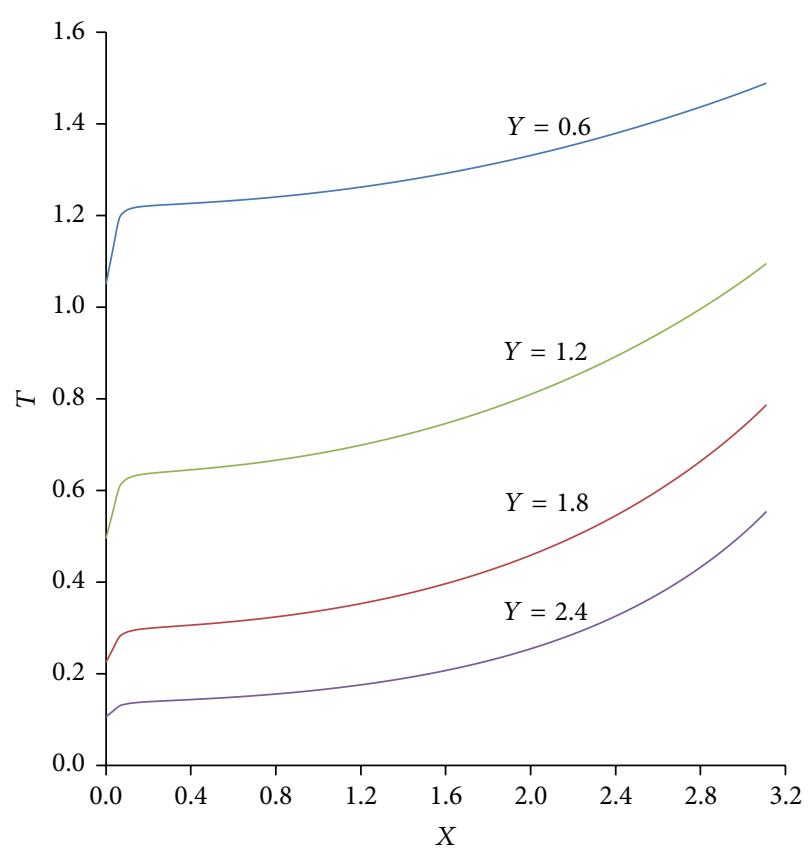

FIgURE 2: Temperature profile against $X$ with $\gamma=0.2, \operatorname{Pr}=0.7$, and $\tau=6.00$.

The temperature profiles against the time $\tau$ at various positions of the radial coordinate $Y$ have been plotted in Figure 4 with $\gamma=0.2$ and at $X=\pi / 6$. The Prandtl number $\operatorname{Pr}=0.7$ is chosen. From Figure 4 , it can be observed that the temperature profile oscillates harmonically near the surface of the cylinder and that there are no more oscillations as we move away from the surface. This is because the oscillating surface temperature makes the temperature of the fluid near the surface oscillate too. At a distance far away from the surface, the fluid temperature does not oscillate frequently because of the influence of the ambient temperature.

The effect of heat generation $\gamma=0.0,0.2,0.4,0.6,0.8$ on the temperature and velocity profiles with $\operatorname{Pr}=0.7$ at $X=\pi / 6$ and $\tau=6.00$ is illustrated in Figures 5 and 6 . Both Figures 5 and 6 show that the increasing values of $\gamma=0.0,0.2,0.4,0.6,0.8$ lead to increases in the temperature and the velocity profiles. The increase in the heat generation parameter causes the thermal boundary layer near the surface, causing the fluid temperature near the surface to increase. As $\gamma$ increases, the increase in the temperature near the surface leads to an increase in the velocity gradient and enhances the fluid velocity. For $\gamma=0.0,0.2,0.4,0.6,0.8$, the maximum values of the velocity are shown to be 0.2720 , $0.3125,0.3532,0.4060$, and 0.4714 , and these occur at $Y=$ 0.84 with the corresponding temperatures of $0.8652,0.9723$, $1.1188,1.3277$, and 1.6241. Thus, it can be observed that the temperature at $Y=0.84$ increases by $75.89 \%$ and the velocity increases by $19.94 \%$ as $\gamma$ increases from 0.0 to 0.8 . At $Y=1.80$, at which the velocity distribution tends to decrease are recorded to be $0.1548,0.1867,0.2197,0.2566$, and 0.2910 corresponding to temperatures of $0.2451,0.3125$, $0.4136,0.5586$, and 0.7443 , so that as $\gamma$ increases from 0.0 to 


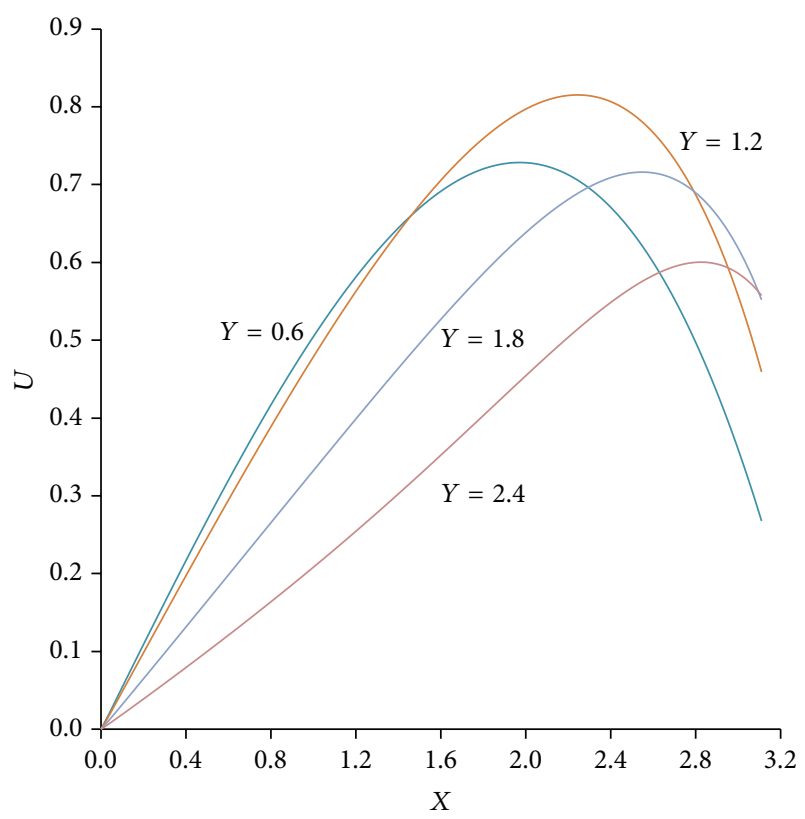

FIgURE 3: Velocity profile against $X$ with $\gamma=0.2, \operatorname{Pr}=0.7$, and $\tau=6.00$.

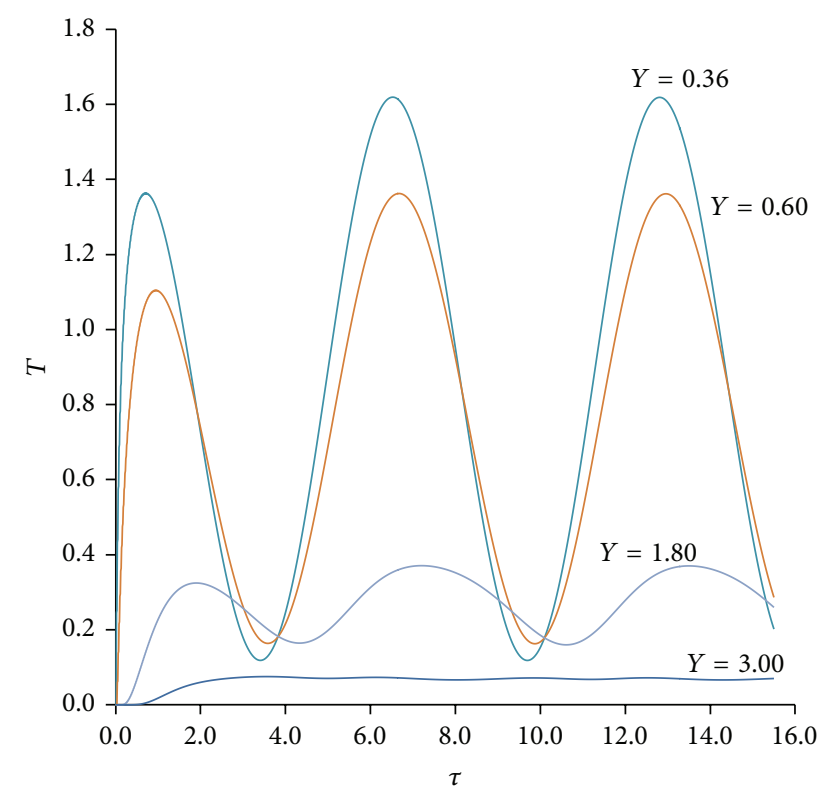

FIGURE 4: Temperature profile against time, $\tau$, with $\gamma=0.2$ and $\operatorname{Pr}=$ 0.7 .

0.8 , the velocity increases by $13.62 \%$ and the temperature by $49.92 \%$.

The local values of the skin friction and the Nusselt number are obtained for different values of the heat generation parameter $\gamma$ on the surface of the circular cylinder against the axial coordinate value $X$ from $(X=0)$ to $(X=\pi)$ at time $\tau=$ 6.28. The numerical results have been illustrated in Figures 7 and 8 , respectively. It can be seen that an increase in the heat generation parameter $\gamma$ leads to a decrease in the local

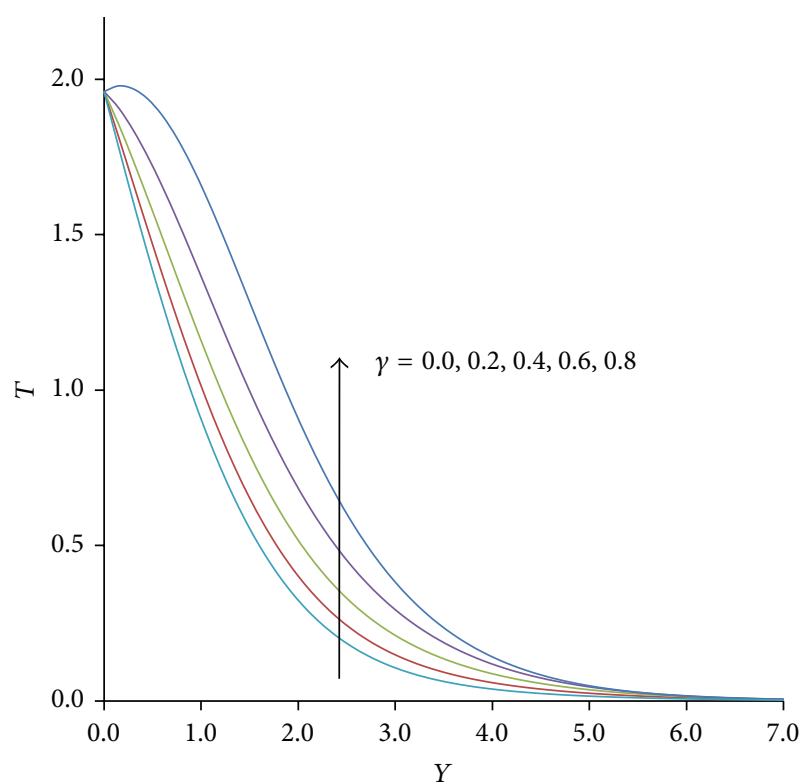

Figure 5: Temperature profile against $Y$ with $\operatorname{Pr}=0.7$ and $\tau=6.00$ at $X=\pi / 6$.

Nusselt number $\mathrm{NuGr}^{-1 / 4}$ which becomes negative for large values of $\gamma$. This is to be expected, since the heat generation mechanism creates a layer of hot fluid near the surface and hence the rate of heat transfer from the surface decreases. This phenomenon was discussed earlier in the section on Figures 5 and 6 as $\gamma$ increases the fluid temperature near the surface increases. Since the fluid temperature near the surface increases, the viscosity of the fluid increases, leading the local skin friction $C_{f} \mathrm{Gr}^{1 / 4}$ to increase.

Figures 9 and 10 present the effect of different values of the Prandtl number $\operatorname{Pr}=0.7,2.0,3.0,7.0$ on the temperature and velocity profiles at $X=\pi / 6$ and $\tau=6.00$ with heat generation $\gamma=0.2$. These figures show that the fluid temperature and velocity distribution tend to be lower for higher Prandtl numbers. This is because, when the value of $\mathrm{Pr}$ is higher, the thermal boundary layer becomes thinner, leading to a decrease in the fluid temperature and in the velocity distribution. The reason for this is that Pr controls the relative thickness of the momentum and thermal boundary layers. As Pr decreases, the heat diffuses more quickly because the thickness of the thermal boundary layer is greater. This means that for gases, for which the Pr range is between 0.7 and 1.0, the thickness of the thermal boundary layer is much greater compared to water, for which the range of Pr is around 7.0 to 8.0. Furthermore, the temperature and velocity profiles decrease and approach zero as the coordinate of $Y$ increases or the distance from the cylinder becomes greater.

Figure 11 shows the effects of the Prandtl number on the local skin friction $C_{f} \mathrm{Gr}^{1 / 4}$ and the heat transfer rate $\mathrm{NuGr}^{-1 / 4}$ when $\gamma=0.2$ at $\tau=6.28$. The local skin friction decreases as Pr increases from 0.7 to 7.0 while the local heat transfer rate increases. Increasing values of Pr will decrease the shear stress because Pr gives thicker velocity profiles; this can be seen in Figure 8. This is the reason why the skin friction decreases. 


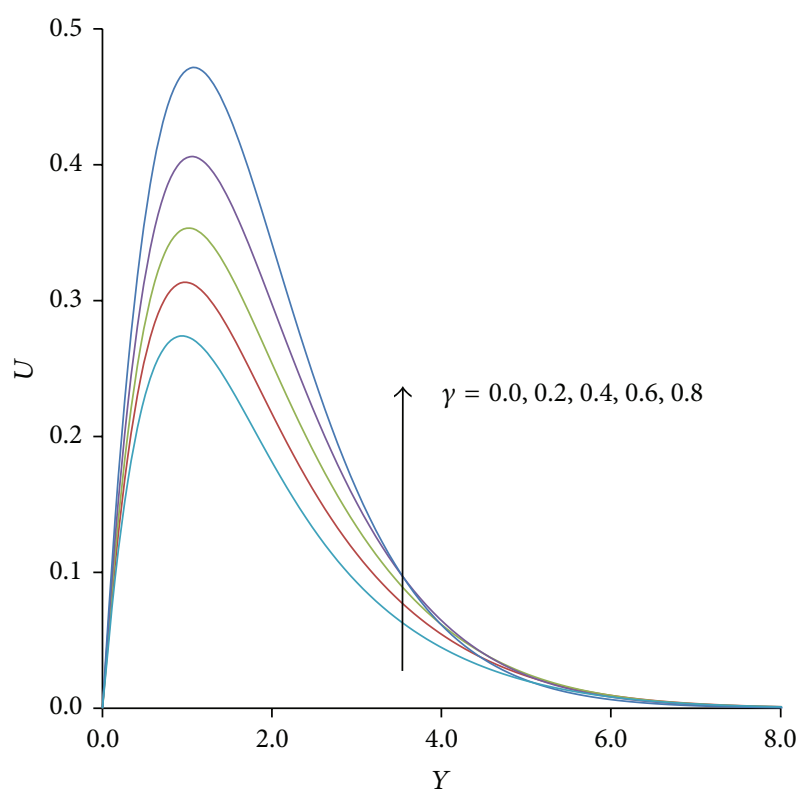

Figure 6: Velocity profile against $Y$ with $\operatorname{Pr}=0.7$ and $\tau=6.00$ at $X=\pi / 6$.

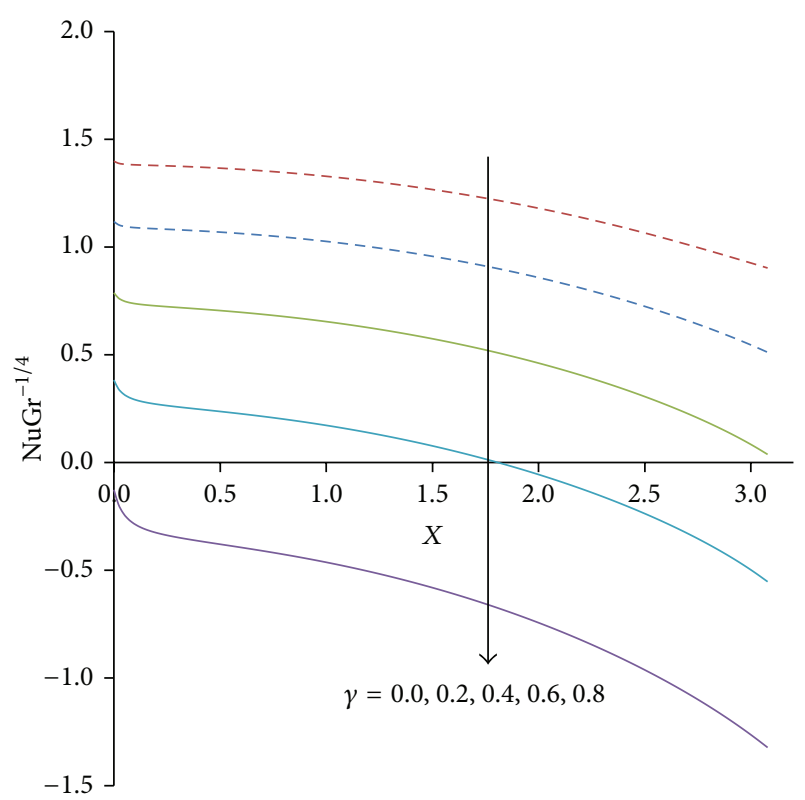

Figure 7: Nusselt number for different values of $\gamma$ with $\operatorname{Pr}=0.7$ at $\tau=6.28$

On the other hand, an increased value of Pr will increase the local heat transfer rate because when Pr becomes larger, the thermal boundary layer becomes thinner and the fluid temperature decreases.

\section{Conclusion}

Oscillatory free convection on a circular cylinder in presence of heat generation has been investigated in this paper with the different values of Prandtl number $\operatorname{Pr}=$

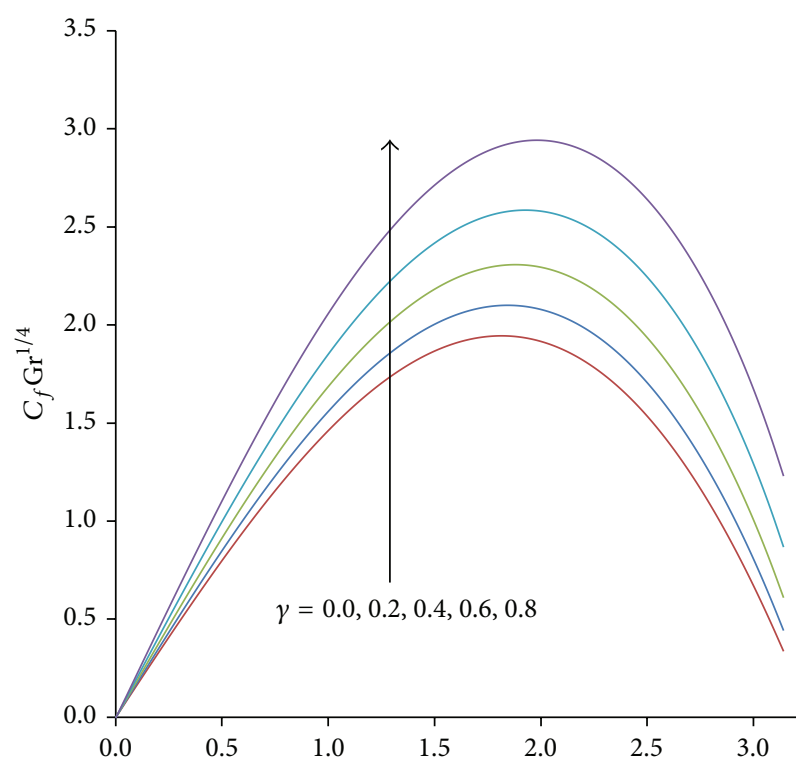

FIGURE 8: Skin friction for different values of $\gamma$ with $\operatorname{Pr}=0.7$ at $\tau=6.28$.

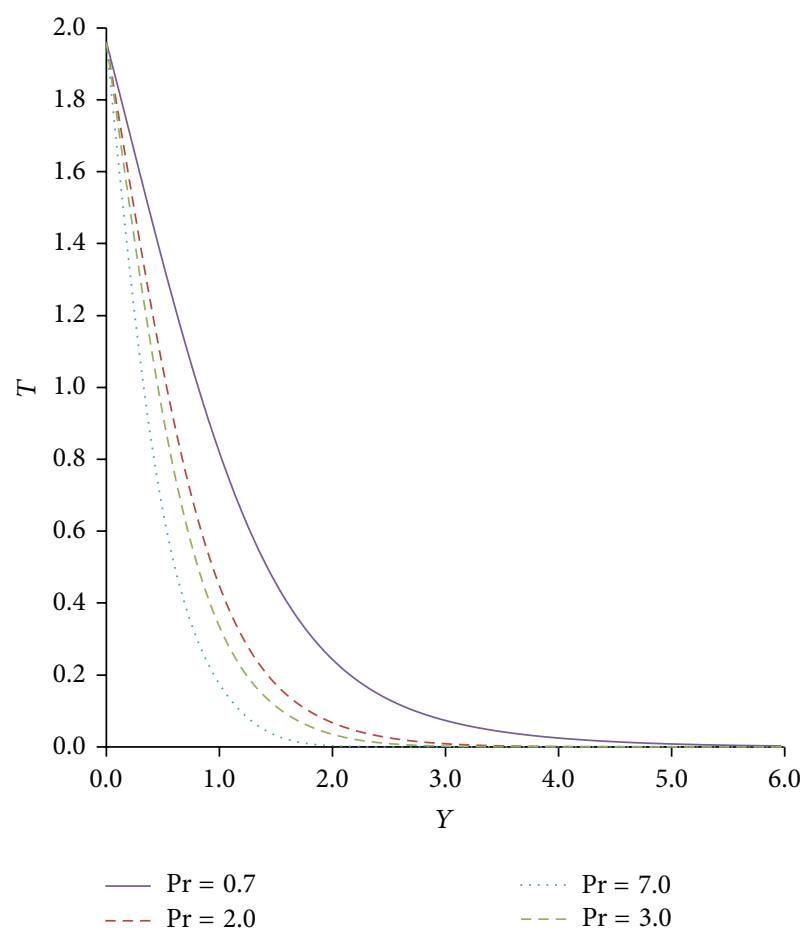

FIGURE 9: Temperature profile against $Y$ with $\gamma=0.2$ and $\tau=6.00$ at $X=\pi / 6$.

$0.7,2.0,3.0,7.0$ and different heat generation parameters $\gamma=$ $0.2,0.4,0.6,0.8$. The governing boundary layer equations were transformed into a nondimensional form, and the resulting nonlinear systems of partial differential equations were solved numerically by an implicit finite difference scheme of Crank-Nicolson type. The solutions are obtained for the entire region of the circular cylinder, from the lower 


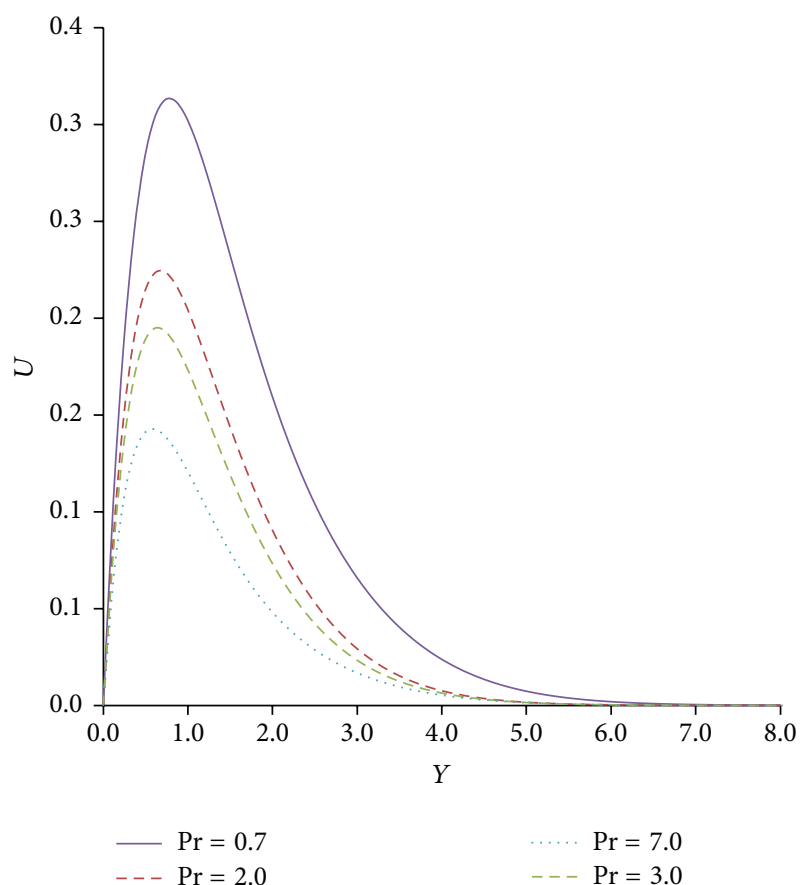

FIGURE 10: Velocity profile against $Y$ with $\gamma=0.2$ and $\tau=6.00$ at $X=\pi / 6$.

stagnation point $(X=0)$ to upper stagnation point $(X=\pi)$. The effect of heat generation with different values of Pr on free convection about a circular cylinder with oscillating surface temperature has been analyzed. From the investigation, the interesting conclusion is summarized as follows.

(1) The effects of heat generation parameter $\gamma$ on velocity and temperature profiles as well as on the skin friction and the heat transfer rate have been investigated. An increase in the values of heat generation parameter $\gamma$ leads to increase both velocity and temperature profiles as well as the skin frictions coefficient but the local rate of heat transfer decreases for $\operatorname{Pr}=0.7$.

(2) The increase in Prandtl number, Pr, with a fixed heat generation parameter leads to a decrease in all the velocity profile, the temperature profile, and the local skin friction coefficient, $C_{f}$, but increases the local rate of heat transfer, $\mathrm{Nu}$.

(3) The steady flow existing around the circular cylinder has been found. The temperature profile oscillates harmonically near the surface of the cylinder and there are no more oscillations as we move away from the surface.

\section{Conflict of Interests}

The authors declare that there is no conflict of interests regarding the publication of this paper.

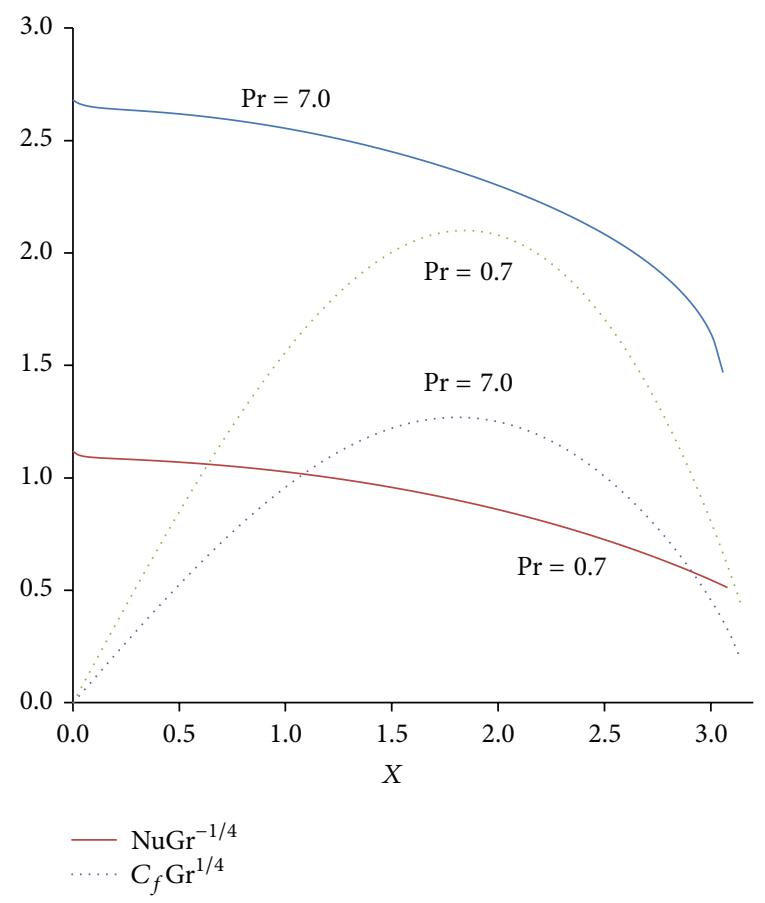

FIGURE 11: Local skin friction, Nusselt number of $\operatorname{Pr}$ with $\gamma=0.2$ at $\tau=6.28$.

\section{Acknowledgments}

The authors would like to acknowledge the financial support received from the University Tun Hussein Onn Malaysia (UTHM) (Fundamental Research Grant Scheme FRGS/2/2010/SG/UTHM/02/4/0761) and the Universiti Kebangsaan Malaysia Grant no. DIP-2012-12.

\section{References}

[1] K. Vajravelu and A. Hadjinicolaou, "Heat transfer in a viscous fluid over a stretching sheet with viscous dissipation and internal heat generation," International Communications in Heat and Mass Transfer, vol. 20, no. 3, pp. 417-430, 1993.

[2] K. Vajravelu, "Natural convection at a heated semiinfinite vertical plate with internal heat generation," Acta Mechanica, vol. 34, no. 1-2, pp. 153-159, 1979.

[3] A. J. Chamkha and C. Issa, "Effects of heat generation/absorption and thermophoresis on hydromagnetic flow with heat and mass transfer over a flat surface," International Journal of Numerical Methods for Heat and Fluid Flow, vol. 10, no. 4, pp. 432-448, 2000.

[4] M. M. Molla, M. A. Hossain, and L. S. Yao, "Natural convection flow along a vertical wavy surface with uniform surface temperature in presence of heat generation/absorption," International Journal of Thermal Sciences, vol. 43, no. 2, pp. 157-163, 2004.

[5] A. Ferdousi and M. A. Alim, "Natural convection flow from a porous vertical plate in presence of heat generation," International University Journal Science Technology, vol. 5, no. 1, pp. 7380, 2010.

[6] M. M. Molla, M. A. Hossain, and M. C. Paul, "Natural convection flow from an isothermal horizontal circular cylinder in 
presence of heat generation," International Journal of Engineering Science, vol. 44, no. 13-14, pp. 949-958, 2006.

[7] M. Mamun Molla, S. C. Paul, and M. Anwar Hossain, "Natural convection flow from a horizontal circular cylinder with uniform heat flux in presence of heat generation," Applied Mathematical Modelling, vol. 33, no. 7, pp. 3226-3236, 2009.

[8] C.-Y. Cheng, "Natural convection heat transfer from a horizontal isothermal elliptical cylinder with internal heat generation," International Communications in Heat and Mass Transfer, vol. 36, no. 4, pp. 346-350, 2009.

[9] M. Miraj, M. A. Alim, and M. A. H. Mamun, "Effect of radiation on natural convection flow on a sphere in presence of heat generation," International Communications in Heat and Mass Transfer, vol. 37, no. 6, pp. 660-665, 2010.

[10] J. H. Merkin, "Oscillatory free convection from an infinite horizontal cylinder," Journal of Fluid Mechanics, vol. 30, pp. 561575, 1967.

[11] A. K. Chatterjee and L. Debnath, "Double boundary layers in oscillatory convective flow," Il Nuovo Cimento B Series 11, vol. 52, no. 1, pp. 29-44, 1979.

[12] P. Ganesan and H. P. Rani, "Transient natural convection along vertical cylinder with Heat and Mass transfer," Heat and Mass Transfer/Waerme- und Stoffuebertragung, vol. 33, no. 5-6, pp. 449-455, 1998.

[13] H. P. Rani and R. Devaraj, "Numerical solution of unsteady flow past a vertical cylinder with temperature oscillations," Forschung im Ingenieurwesen/Engineering Research, vol. 68, no. 2, pp. 75-78, 2003.

[14] M. K. Jaman and M. A. Hossain, "Effect of fluctuating surface temperature on natural convection flow over cylinders of elliptic cross section," Open Transport Phenomena Journal, vol. 2, no. 1, pp. 35-47, 2010.

[15] R. L. Verma and P. Singh, "The effect of plate oscillations on horizontal free convection flow," Australian Journal of Physics, vol. 30, no. 3, pp. 335-345, 1977.

[16] M. A. Hossain, S. K. Das, and I. Pop, "Heat transfer response of MHD free convection flow along a vertical plate to surface temperature oscillations," International Journal of Non-Linear Mechanics, vol. 33, no. 3, pp. 541-553, 1998.

[17] V. R. Prasad, B. Vasu, O. Anwar, and R. Parsha, "Unsteady free convection heat and mass transfer in a Walters- $B$ viscoelastic flow past a semi-infinite vertical plate: A Numerical Study," International Journal of Thermal Sciences, vol. 15, pp. 291-305, 2011.

[18] V. R. Prasad, N. B. Reddy, and R. Muthucumaraswamy, "Radiation and mass transfer effects on two-dimensional flow past an impulsively started infinite vertical plate," International Journal of Thermal Sciences, vol. 46, no. 12, pp. 1251-1258, 2007.

[19] Z. Udin and M. Kumar, "Unsteady free convection in a fluid past an inclined plate immersed in a porous medium," Computer Modelling and New Technologies, vol. 14, pp. 41-47, 2010.

[20] B. Carnahan, H. A. Luther, and J. O. Wilkes, Applied Numerical Methods, John Wiley \& Sons, New York, NY, USA, 1969.

[21] A. R. M. Kasim, M. A. Admon, and S. Shafie, "Free convection boundary layer flow of a viscoelastic fluid in the presence of heat generation," World Academy of Science, Engineering and Technology, vol. 75, pp. 492-499, 2011. 


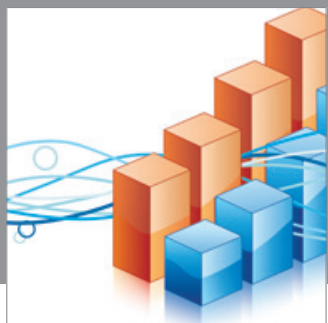

Advances in

Operations Research

mansans

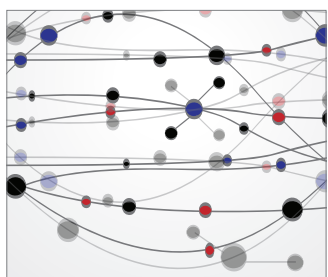

The Scientific World Journal
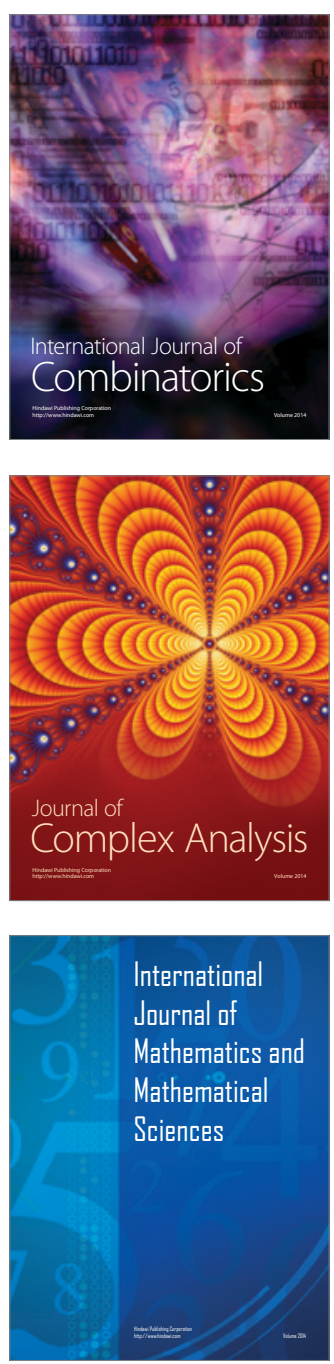
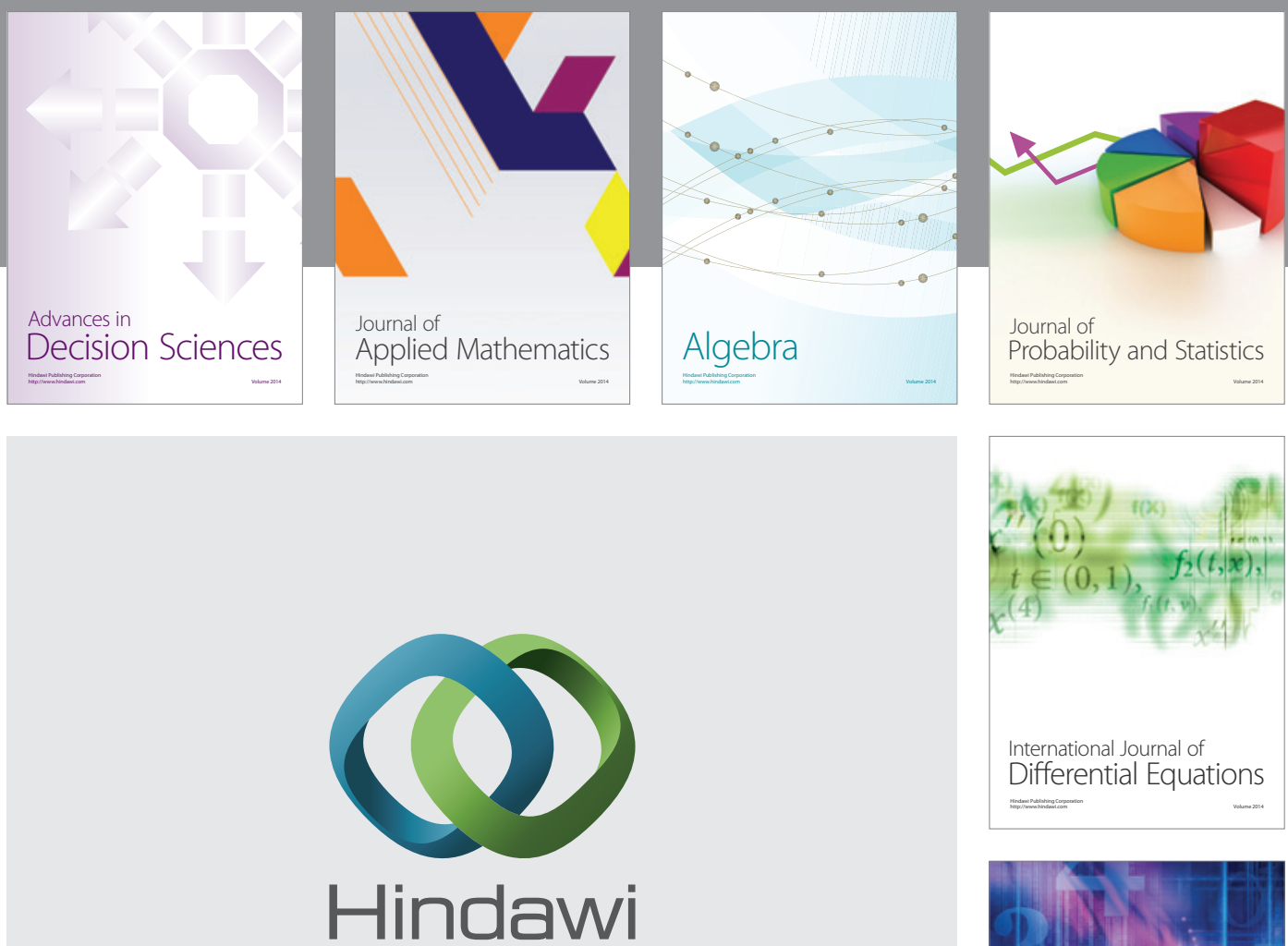

Submit your manuscripts at http://www.hindawi.com
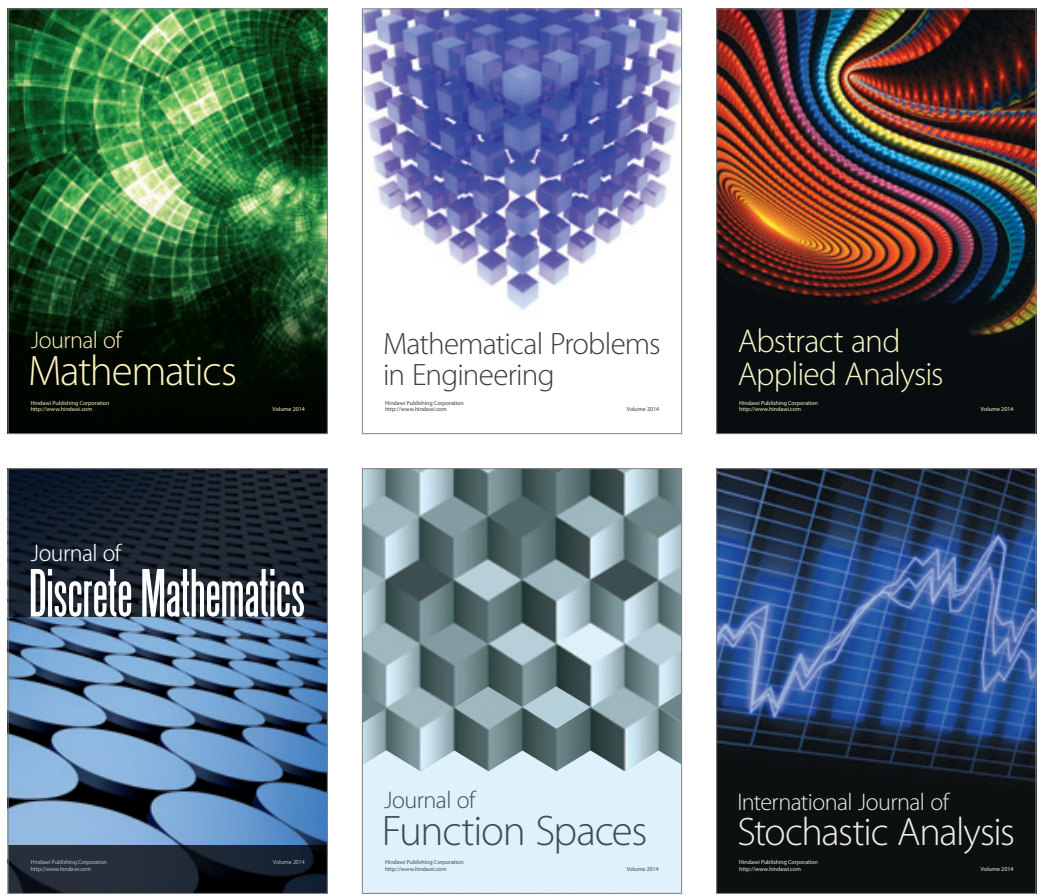

Journal of

Function Spaces

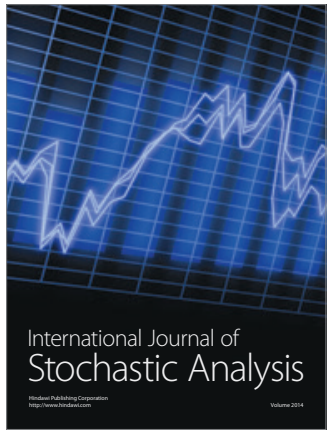

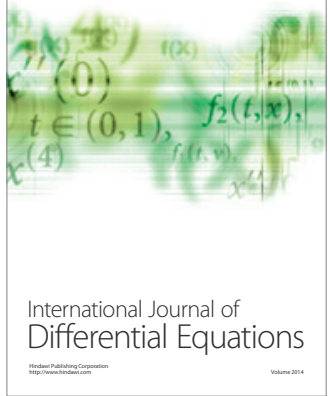
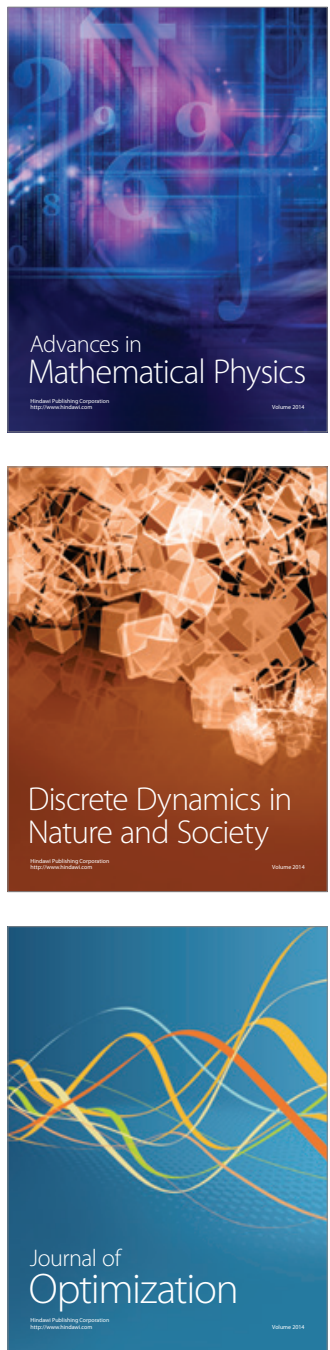